\title{
Effect of metallicity on low mass X-ray binaries in globular clusters
}

\author{
Natalia Ivanova ${ }^{1}$ \\ ${ }^{1}$ Physics and Astronomy Department, Northwestern University, 2145 Sheridan Rd, Evanston \\ IL 60208 \\ email: nata@northwestern.edu
}

\begin{abstract}
We propose that the observed difference in the formation rates of bright low-mass X-ray binaries in metal-rich and metal-poor globular clusters can be explained by taking into account the difference in the stellar structure of main sequence donors with masses between $\sim 0.85 M_{\odot}$ and $\sim 1.25 M_{\odot}$ at different metallicities. This difference is caused by the absence of an outer convective zone in metal-poor main sequence stars in this mass range. In the result, magnetic braking, a powerful mechanism of orbital shrinkage, does not operate and dynamically formed main sequence - neutron star binaries fail to start mass transfer or appear as bright low-mass X-ray binaries.
\end{abstract}

Keywords. binaries: close - X-rays: binaries - globular clusters:general - stellar dynamics.

\section{Observational motivations}

Bright low-mass X-ray binaries (LMXBs) in globular clusters (GCs) of our Galaxy and of M31 preferentially reside in metal-rich GCs (Grindlay 1993, Belazzini et al. 1995). Recent extragalactic observations revealed the same tendency: in NGC 4472 (Kundu et al. 2002, Maccarone et al. 2004) and in M87 (Jordán et al., 2004) metal-rich GCs are $3 \pm 1$ times as likely to contain bright LMXBs as metal-poor GCs.

In detail, from observations of galactic GCs, two currently known GC-residing persistent LMXBs with a possibly non-degenerate (but also not a subgiant) companion are contained only in metal-rich clusters (Verbunt \& Lewin 2005), as well as two known GCcontained luminous transient LMXBs with a possibly non-degenerate companion. Five persistent bright LMXBs are observed in relatively metal-poor clusters. Four of them show indications of being ultracompact and one is likely to be a subgiant according to its orbital period. One luminous transient LMXB in a metal-poor cluster is identified as an ultracompact, and for the bright LMXB in Terzan 1 (metal-poor), there is no information on whether it is ultracompact or not.

This data set hints that possibly bright LMXBs consisting of a NS and a main sequence (MS) star can preferably be formed in metal-rich clusters.

\section{Dynamically formed NS-MS binaries}

NS-MS binaries could form via exchange encounters or via tidal captures. In the first case, the minimum period of the post-exchange binary is limited both by the period at contact for a given eccentricity and by the period determined by the binary energy conservation during the encounter. Due to the energy conservation, when NS replaces a less massive star, the binary separation in the formed post-exchange binary is larger than the binary separation in the pre-exchange binary. 
The binary periods of tidally captured binaries are limited at the upper limit by the closest approach at which tidal interactions are still strong enough to make a bound system. The lower limit is determined by the closest approach at which a MS star will pass and still will not be destroyed. The parameter space for tidally captured binaries where the MS star does not overfill its Roche lobe at the closest approach is very restricted.

\section{Successful LMXBs Candidates}

NS-MS binaries are evolved under the influence of gravitational radiation, magnetic braking and tides. In metal-poor clusters, only stars $\leqslant 0.85 M_{\odot}$ have the developed outer convective zone - only there magnetic braking and effective convective tides operate. This determines the maximum initial binary period, for different eccentricities, such that the binary will start the mass transfer (MT) before the MS star leaves the MS or another dynamical encounter occurs.

As a result, the parameter-space available for post-exchange binaries that can successfully start MT in metal-rich clusters is substantially larger than in metal-poor clusters. Also, in metal-poor clusters, the efficiency of tidal captures is significantly reduced for MS stars with radiative envelopes $\left(\geqslant 0.85 M_{\odot}\right)$ and only MS stars with masses less than $0.55 M_{\odot}$ can be formed via tidal capture without overfilling their Roche lobe during the event.

\section{Persistent or transient?}

In metal-poor clusters, all MT NS-MS binaries with a MS star $\geqslant 0.85 M_{\odot}$ will evolve on the time-scale predicted by gravitational radiation until the donor decreases its mass enough to develop the deep outer convective zone. Before this moment, the MT binary appears as a transient X-ray source. When the deep outer convective zone is developed, the MT rates jumps and an LMXB can become persistent only for a very short period of time.

In metal-rich clusters, an LMXB appears first as a persistent LMXB. When the donor decreases its mass to $\sim 0.7 M_{\odot}$, the MT rate becomes very close to critical and remains close for a long time. Even a slight discrepancy in the value of $\dot{M}_{\text {crit }}$ leads to large differences in how much time an LMXB spend as persistent $\tau_{\text {pers }}$ or as a transient system $\tau_{\text {tr }}$. This affects how many qLMXBs and how many bright LMXBs can be present in metal-rich clusters.

\section{Acknowledgements}

This work is supported by a Chandra Theory Award to N. Ivanova.

\section{References}

Bellazzini, M., Pasquali, A., Federici, L., Ferraro, F. R., \& Pecci, F. F. 1995, ApJ, 439, 687

Grindlay, J. E. 1993, ASP Conf. Ser. 48: The Globular Cluster-Galaxy Connection, 48, 156 Jordán, A., et al. 2004, ApJ, 613, 279

Kundu, A., Maccarone, T. J., \& Zepf, S. E. 2002, ApJL, 574, L5

Maccarone, T. J., Kundu, A., \& Zepf, S. E. 2004, ApJ, 606, 430

Verbunt, F. \& Lewin, W., in press, Chapter 8 in "Compact Stellar X-ray Sources," eds. W.H.G. Lewin and M. van der Klis, Cambridge University Press. 